\title{
Evaluating the Time Dependent Anti-plasmodium Activity of Andrographolide and Chloroquine on Different Stages of the Intraerythrocytic Cycle of Plasmodium Falciparum 3D7 in Vitro
}

ashraf Ahmad Issa alapid

University Putra Malaysia

Zaid 0. Ibraheem

Al-Rafidain University College

\section{Ramatu Omenesa Bello}

Ahmadu Bello University Faculty of Pharmaceutical Science

Intan Safinar Ismail

University Putra Malaysia Institut Biosains

Ngah Zasmy Unyah

University putra malaysia

Abdullahi Sharif Alhassan

Bayero University Kano

Norshariza Nordin

University Putra Malaysia: Universiti Putra Malaysia

\section{Mohammed Nasiru Wana}

Abubakar Tafawa Balewa University

Basir Rusliza ( $\sim$ rusliza@upm.edu.my )

University Putra Malaysia https://orcid.org/0000-0003-3363-6630

\section{Roslaini Abd. Majid}

National Defence University of Malaysia

\section{Research}

Keywords: Anti-plasmodium, Chloroquine, Andrographolide, P. falciparum, pLDH and Intraerythrocytic

Posted Date: November 13th, 2020

DOI: https://doi.org/10.21203/rs.3.rs-104897/v1

License: (c) (i) This work is licensed under a Creative Commons Attribution 4.0 International License. Read Full License 


\section{Abstract}

Background: The increasing incidence of drug resistance among various strains of Plasmodium falciparum has compelled researchers to search for new improved therapeutic alternatives to current antimalarials. Consequently, the study aimed to investigate the effect of varying the duration of andrographolide exposure on its antiplasmodial effect against intra erythrocytic stages of the $P$. falciparum 3D7 parasite. Although andrographolide has demonstrated prior anti-plasmodial effect against $P$. falciparum 3D7, its time-dependent effect subsequent to different durations of drug exposure in addition to the impact of relevant pharmacologically active concentrations on the cellular morphology of various intraerythrocytic stages of the $P$. falciparum 3D7 parasite cycle are limited.

Methods: P. falciparum 3D7 parasites cultivated in vitro in blood cultures were individually incubated with different concentrations of andrographolide, chloroquine and drug-free parasite culture which served as the representative control. Suppression of parasite growth was determined by parasite lactate dehydrogenase (pLDH) based drug sensitivity assay. The inhibition of parasite growth and changes in morphology of intraerythrocytic parasites subsequent to treatment initiation with andrographolide or chloroquine were assessed upon commencement of a synchronized cycle at 12, 24 and $48 \mathrm{~h}$ respectively.

Results: Andrographolide showed satisfactory growth inhibitory effect however its inhibitory activity was substantially lower when compared to that of chloroquine. Unlike chloroquine which showed maximal inhibitory activity within the first $12 \mathrm{~h}$ of the cycle, suppression of parasite growth by andrographolide was most prominent during the development of early trophozoites (viz the second 12 hours). Andrographolide failed to produce any effect on the morphology of ring stage parasites, it however produced a noticeable change in the morphological appearance and sizes of mature trophozoites. Whereas, with chloroquine notable changes to ring and trophozoite stages of the parasites were evident.

Conclusion: The data obtained indicates the potential role of andrographolide as an adjunctive treatment in malaria subject to further clinical evaluations.

\section{Background}

Malaria infection is a leading cause of morbidity and mortality in tropical and subtropical regions of the world including Africa, Southeast Asia, and Eastern Mediterranean regions [1]. Infection is caused by any of the five known plasmodia that infect humans namely Plasmodium vivax, P. falciparum, P. malariae, $P$. ovale or the more recently characterized simian malaria parasite $P$. knowlesci [2]. Over the past few decades, significant resurgence of malaria cases and associated mortality have been reported despite judicious implementation of rational eradication programmes [1]. The malaria situation is hypothesized to have deteriorated commensurately with the emergence of multi drug resistant strains of the plasmodium parasite.

Hitherto, there are a few classes of drugs used to treat malaria infection. The various classes of anti-malarial drugs act against differecnt stages of the Plasmodium parasite's life cycle [3] viz the sporozoites, hepatic schizonts or stages of the intraerythrocytic cycle. Generally, sporocytocidal drugs are given as prophylactic agents against malaria infection while blood schizontocidal drugs, including, chloroquine (CQ), quinine, mefloquine, primaquine, proguanil (chloroguanide) and pyrimethamine are given during the intraerythrocytic cycle which is a crucial stage targeted for parasite eradication and for arresting the progression of disease [4]. Aside from their schizonticidal properties chloroquine (CQ), 8-aminoquinolines are also utilized to reduced or prevent reoccurrence of relapsing 
malaria caused by P. vivax or P. ovale [5]. Both of the afcorementioned species have hepatic schizonts that may persist for long intervals of time intra-hepatically [6].

The discovery of novel antimalarial principles from plant sources in the past coupled with the emergence of drugresistance among species of $P$. falciparum to current antimalarials has prompted researchers to extensively explore phytochemicals as potential sources of anti-malarial pharmacophore candidates against antigenically variant malaria parasites as potential substitutes for the conventional anti-malarial drugs. Ethnomedicinal plants offer potential as a source of viable cost-effective anti-malarial principles in the realm of malaria chemotherapy particularly in underprivileged populations where access to healthcare facilities is limited.

Andrographolide (AG) is a labdane diterpenoid derivative present abundantly in the herbaceous plant Andrograpcchis paniculata which is extensively cultivated in Southern Asia, China and some parts of Europe [7]. $\mathrm{AG}$ is an active principle of $A$. paniculate, and several publications have appeared in recent years highlighting the biological characteristics of AG including its antimicrobial, anti-inflammatory and antioxidant properties $[8,9]$.

The in vitro and in vivo anti-plasmodial activity of andrographolide was screened previously by Mishra et al., [10]. Additionally, Zaid et al., (2015) [11] reported the probable mechanism of action of AG as via permeation pathways channels visible on the membrane of infected RBCs as well as its impact on merozoite invasion. However, the impact of $A G$ against different stages of the plasmodium parasite has not been sufficiently explored.

This study aimed to determine the time-dependent effect(s) of AG and CQ on the various stages of the $P$. falciparum 3D7 parasite's intraerythrocytic cycle (as a means to assess the stage of the parasite's reproductive cycle wherein maximum antiparasitic effect can be achieved) in addition to its impact on size and morphology of parasite forms (variants) constituting the intraerythrocytic cycle.

\section{Methods}

\section{Red blood cells}

Uninfected RBCs (type 0- negative) was donated by the first author under the supervision of a haematologist. The blood was mixed with citrate phosphate buffer as anticoagulant (in the ratio of 1:9 anticoagulant/ blood); the blood was subsequently washed thrice using washing medium to remove plasma and white blood cells and resuspended to obtain a suspension of RBCs. The washing medium contained RPMI-1640, 25 mM HEPES (4-(2hydroxyethyl)-1-piperazine-ethan-sulphonic acid) buffer ( $\mathrm{pH}$ 7.4), $24 \mathrm{mM}$ sodium bicarbonate, $11 \mathrm{mM}$ glucose and $50 \mu \mathrm{g} / \mathrm{L}$ gentamicin. The standard protocol for blood washing was adopted as previously described [12].

\section{Chemicals and consumables}

Human 0- erythrocytes were pelleted from blood suspended in RPMI-1640 medium. Albumax II was procured from Gibco BRL (Grand Island, NY, USA). Whereas, HEPES, triton X-100, EDTA, saponin, sorbitol, hypoxanthine, (100X) phosphate buffered saline (PBS), Giemsa stain and chloroquine diphosphate (CQ) were purchased from SigmaAldrich (St. Louis, MO, USA). Gentamicin was purchased from (Jiangxi Dongxu Chemical Technology Co., Ltd) while AG was purchased from Indofine Biochemical company Inc. (Cat No.: A-003).

\section{Parasite}


P. falciparum 3D7 (a CQ sensitive strain) was procured as cryopreserved specimens (at $-160{ }^{\circ} \mathrm{C}$ ) under liquid nitrogen from the Department of Medical Microbiology and Parasitology, Faculty of Medicine, Universiti Malaya, Kuala Lumpur, Malaysia. The cryovials were thawed at $37^{\circ} \mathrm{C}$ using the standard thawing technique for malaria cryopreserved specimens. The parasites were subsequently mixed with the RBCs suspension at a haematocrit of $2 \%-5 \%$ and cultured per standard cultivation conditions viz micro-aerophilic environment $\left(5 \% \mathrm{CO}_{2}, 37^{\circ} \mathrm{C}\right)$. Recovery of the cryopreserved parasites was carried out, using the modified protocol described by Trager and Jensen [13].

\section{Drug sensitivity assay and time-dependent effect}

Malaria drug sensitivity assay was carried out using Plasmodium Lactate DeHydrogenase-Technique (pLDH) as described by Makler et al., [14]. Stock solutions of $10 \mathrm{mM} \mathrm{AG}$ and CQ were prepared in DMSO and PBS $(\mathrm{pH}=7.4)$ respectively. Fifty microliters $(50 \mu \mathrm{L})$ of the synchronized culture was loaded into flat-bottomed 96 -well microtiter plates featuring different concentrations of each drug (1 $\mathrm{nM}$ to $1 \mathrm{mM})$. The mixtures were prepared in triplicate and incubated at standard conditions for parasite cultivation over different time intervals; $12 \mathrm{~h}, 24 \mathrm{~h}$, and 48 hours post-cultivation. The final parasitemia and hematocrit were maintained at $1 \%$ with parasitized RBCs (PRBCs) synchronized at the ring stage. The plates were deep frozen at $-80^{\circ} \mathrm{C}$ for 1 hour after incubation and parasite growth was screened using Malstat reagent mixed with NBT/PES reagent (4:1 Malstat, NBT/PES) [14]. The sensitivity of the parasite to either AG or CQ was estimated through extrapolation of the log (dose) response curve. The concentrations required to inhibit the parasite growth at $20 \%, 50 \%$, and $90 \%\left(I C_{20}, I C_{50}\right.$, and $\left.I C_{90}\right)$ respectively were identified.

\section{Parasite cultivation and maintenance}

The procured parasites were cultivated in a culture suspension containing O- negative RBCs and suspended in a complete RPMI-1640 malaria culture medium (CMCM) containing 25 mM HEPES, 0.75 mM hypoxanthine, Albumax 5\%, $24 \mathrm{mM}$ sodium bicarbonate, $11 \mathrm{mM}$ glucose, and $20 \mu \mathrm{g} / \mathrm{ml}$ gentamicin at $\mathrm{pH}$ and hematocrit levels of 7.4 and $2 \%$, respectively. Subsequently, the culture was incubated at $37{ }^{\circ} \mathrm{C}$ in a micro-aerophilic environment containing $\left(5 \% \mathrm{CO}_{2}\right)$. The medium was changed daily, and parasite growth was monitored using Giemsa stained thin blood smears. The parasites were passed to other culture media upon attaining a threshold parasitaemia of $2 \%$.

\section{Parasite synchronization}

The process of culture (parasite) synchronization at the ring stage of the intraerythrocytic cycle was carried out prior to the drug sensitivity assay. The process of synchronization was performed using the sorbitol synchronization technique as described by Lambros and Vanderberg [15]. Briefly, the pelleted PRBCs were incubated with sorbitol for 5 min and then cultivated as described above (section Parasite cultivation and maintenance) after washing out the sorbitol solution [15].

Effects of andrographolide and chloroquine on cell morphology and intraerythrocytic cycle progression of $\mathrm{P}$. falciparum 3D7

The impact of $A G$ and $C Q$ on the development of various stages of the $P$. falciparum 3D7 parasite's intraerythrocytic cycle was performed by exposing the synchronized PRBCs to each drug at its $I C_{20}, I_{50}$ and IC 90 concentrations for a period of 48 hours in $75 \mathrm{~cm}^{2}$ cell culture flasks at the standard cultivation conditions as described above (section parasite cultivation and maintenance). The flasks were prepared in triplicate, and the haematocrit (Hct) was maintained at 2\%. During the incubation, inoculums of the cultures were taken after 12, 24 
and 48 hours, respectively. The parasite growth for each of the AG and CQ exposed flasks was compared with that of the non-exposed flasks representing the untreated control. Giemsa-stained thin blood smears were used to monitor the impact of each concentration on the cell count of each stage of the intraerythrocytic cycle as well as the impact against cell size and cell morphology [16]. The percentage of parasitized cells was determined within 2000 PRBCs with the aide of an optical microscope. The percentage of each stage of the life cycle was determined amongst infected cells while the impact of $C Q$ or AG against cell morphology and size measurements were determined by image analysis (Olympus BX51, Olympus Corporation, Tokyo, Japan).

\section{Statistical analysis}

The time-dependent effect of each drug through the span of one complete intraerythrocytic cycle was calculated using GraphPad Prism 6 Software version 6.01 (Inc., La Jolla, CA, USA). The results obtained were expressed as mean \pm S.E.M. Significant differences between treatment groups was determined using One-Way ANOVA followed by a Bonferroni post-hoc analysis for inter groups' comparison using SPSS software version 23. A value of $P<0.05$ was considered significant.

\section{Results}

Drug sensitivity assay and time-dependent effect

As shown in Table 1, AG produced moderate growth inhibitory activity at $I C_{20}, I_{50}$ and $I C_{90}$ which was significantly less than that of CQ $(P<0.05)$ at similar concentrations. Regardless of the slight growth inhibitory effect observed for $A G$ during the initial $12 \mathrm{~h}$ of the cycle (evidenced by significantly higher values of growth inhibitory parameters at $\mathrm{IC}_{20}, \mathrm{IC}_{50}$ and $\mathrm{IC}_{90}$ respectively), inhibition of parasite growth was significantly diminished 24 hours after commencement of the synchronized cycle as shown in Table 1. There was a further albeit marginal decrease in growth inhibitory parameters $\left(\mathrm{IC}_{20}, \mathrm{IC}_{50}\right.$ and $\left.\mathrm{IC}_{90}\right)$ after 48 hours. Although this decline was insignificant as compared to that after 24 hours $(P<0.05)$, it remained significant compared to AG-mediated growth inhibition after 12 hours $(P<0.001)$.

The discrepancy in growth inhibitory activity of CQ along the different time points assessed was less when compared to that of AG where its maximal parasite growth inhibitory activity was observed during the first 12 hours of the cycle and gradually decreased thereafter. Inhibition of parasite growth by CQ after 24 and 48 hours was slightly greater than that after 12 hours as indicated by the decrease in values of the growth inhibitory parameters at 24 and 48 hours of the cycle (Table 1). For CQ, the values at 24 and 48 hours were significantly different $(P<0.05)$ as compared to that after 12 hours (Table 1$)$.

\section{Effects on the intraerythrocytic cycle progression of P. falciparum 3D7 following exposure to andrographolide and chloroquine}

The findings showed that $\mathrm{CQ}$ at $\mathrm{IC}_{50}$ concentration prompted a parasiticidal effect wherein approximately $50 \%$ of the parasites were killed within the first $12 \mathrm{~h}$ of the cycle (Fig. 1B). Moreover, a high frequency of pyknotic cells (dead parasites) and inviable cells were observed after CQ exposure. However, AG failed to produce noticeable parasiticidal effect during this interval and the parasite development sustained a normal course which was comparable to that of the control (Fig. 1A). After $24 \mathrm{~h}$ of the cycle, AG showed prominent parasiticidal effect (Fig. 1C). This finding was in accordance with results of the drug sensitivity and time-dependent effect, wherein the inhibitory growth parameters of AG were prominently decreased after $24 \mathrm{~h}$ of the cycle as compared with those 
after $12 \mathrm{~h}$ (Fig. 1C). Nevertheless, along with CQ exposure, there were incidences of new cell death were absent and the total amount of viable cells remained the same as that after $12 \mathrm{~h}$. In addition, the rings that had been retrieved after $12 \mathrm{~h}$ had transformed into new trophozoites (Fig. 1B). At the end of the cycle (after $48 \mathrm{~h}$ ), there were no dead parasites identified. The overall parasitemia remained the same as compared to that after $12 \mathrm{~h}$ and $24 \mathrm{~h}$ following commencement of the cycle for both $C Q$ and $A G$, respectively (Fig. 1B and 1C).

However, a dominance of schizonts was observed in all the flasks with a total percentage less than that of the control. Furthermore, the number of trophozoites retrieved in the treated flasks was significantly decreased as compared to the control (Fig. 1A). At this stage, new rings appeared to initiate a new cycle. Their number was comparatively less in the chloroquine-treated flasks, indicating a possible role for chloroquine against merozoite rupture or ring invasion. This effect was absent when the parasite was exposed to AG (Fig. 1C).

Table 1

Growth inhibitory parameters $\left(\mathrm{IC}_{20}, \mathrm{IC}_{50}\right.$ and $\left.\mathrm{IC}_{90}\right)$ of andrographolide and chloroquine on P. falciparum 3D7

\begin{tabular}{|c|c|c|c|c|c|c|}
\hline \multicolumn{7}{|c|}{ Growth inhibitory parameters of andrographolide and chloroquine } \\
\hline & $\mathrm{IC}_{20} \mathrm{nM}$ & & $\mathrm{IC}_{50} \mathrm{nM}$ & & $\mathrm{IC}_{90} \mathrm{nM}$ & \\
\hline & andrographolide & chloroquine & andrographolide & chloroquine & andrographolide & chloroquine \\
\hline $\begin{array}{l}12 \\
\text { hours }\end{array}$ & $\begin{array}{l}13200.88 \pm \\
1174.08^{\star \star \star a a a a}\end{array}$ & $9.43 \pm 1.6$ & $\begin{array}{l}38691.75 \pm \\
359.52^{\star \star \star \$ \$ \$ a a a ~}\end{array}$ & $\begin{array}{l}54.44 \pm \\
2.41^{\star}\end{array}$ & $\begin{array}{l}15747.66 \pm \\
165.94^{\star \star \star \$ \$ \$ a a a ~}\end{array}$ & $\begin{array}{l}227.38 \pm \\
13.66^{* \$}\end{array}$ \\
\hline $\begin{array}{l}24 \\
\text { hours }\end{array}$ & $\begin{array}{l}2367.39 \pm \\
76.30^{*} \text { aaa }\end{array}$ & $6.89 \pm 0.79$ & $\begin{array}{l}7983.78 \pm \\
267.82^{\text {aaa }}\end{array}$ & $\begin{array}{l}33.94 \pm \\
1.30\end{array}$ & $\begin{array}{l}57223.87 \pm \\
1494.33^{\text {aaa }}\end{array}$ & $\begin{array}{l}102.90 \pm \\
2.09\end{array}$ \\
\hline $\begin{array}{l}48 \\
\text { hours }\end{array}$ & $\begin{array}{l}587.80 \pm \\
52.01^{\text {aaa }}\end{array}$ & $7.43 \pm 1.22$ & $\begin{array}{l}4148.61 \pm \\
412.62 \text { aaa }\end{array}$ & $\begin{array}{l}20.19 \pm \\
1.79\end{array}$ & $\begin{array}{l}63839.38 \pm \\
6595.98^{\text {aaa }}\end{array}$ & $\begin{array}{l}49.64 \pm \\
1.42\end{array}$ \\
\hline
\end{tabular}

$\left(\mathrm{IC}_{20}, \mathrm{IC}_{50}\right.$ and $\left.\mathrm{IC}_{90}\right)$ (the concentrations required to inhibit the growth of $P$. falciparum 3D7 cultivated in blood culture at the standard conditions by 20,50 and $90 \%$ respectively). Results were expressed as (mean in nM $\pm S E M$ ). $\star_{,}^{* *} \&{ }^{* * *}$ and $\$, \$ \$ \& \$ \$$ represent a statistically significant difference in the parameter as compared to time 48 and 24 , respectively for each drug. Meanwhile, a, aa and aaa represents a statistically significant difference as compared with a cohort parameter of chloroquine with $(P<0.05),(P<0.01)$ and $(P<0.001)$ respectivel Effects of andrographolide and chloroquine on the cell morphology of $P$. falciparum 3D7 Ring stage morphology after 12 hours Unlike CQ, AG failed to produce observable effect(s) on the morphology of ring stage parasites. All ring stage parasites in andrographolide-treated flasks were morphologically comparable to those of the control group (Fig. 2). Furthermore, the sizes of ring stage parasites did not differ significantly as compared to those of the control group (Fig. 3A). On the other hand, a large proportion of ring stage parasites in the chloroquine-treated flasks appeared to have altered morphology observable within the first $12 \mathrm{~h}$ of the cycle. Morphological changes observed included prominent shrinkage, fragmented structure, and loss of cellular integrity. These observations are concordant with the effects of chloroquine against the ring count. Ring count was affected by CQ but not by AG (Figs. 2 and $3 A$ ).

Trophozoite morphology after 24 hours

Both CQ and AG produced prominent changes in the morphological appearance and sizes of mature trophozoites. Morphological changes were apparent during the $12 \mathrm{~h}$ interval between the 12th and 24th hour of the parasite's (synchronized) reproductive cycle. The effect on trophozoite morphology due to CQ was more prominent 
compared to AG as evidenced by the higher numbers of disfigured and shrunken cells observed in chloroquinetreated flasks (Figs. 2 and 3B).

Schizont morphology after 48 hours

Changes in the sizes and morphological appearance of schizonts were minimal to absent when exposed to either $\mathrm{CQ}$ or $\mathrm{AG}$ in the various samples assessed (Figs. 2 and $3 \mathrm{C}$ ).

\section{Discussion}

Recent progress in antimalarial drug research has prompted the search for potent chemotherapeutic agents within the phytochemical sanctuary, particularly following the discovery of artemisinins; a potent antimalarial phytochemical discovered in Artemisia annua [17]. Artemisinin is now recognized as one of the conventional antimalarials, moreover Artemisinin-based combination therapy (ACTs) is now the front-line treatment for $P$. falciparum instead of mono-therapies, especially in the regions wherein multidrug resistant strains of $P$. falciparum persist [18].

It is worthy of mention that several phytochemicals have been investigated for their anti-plasmodial activity in the past, however, the majority of these phytochemicals failed to produce considerable antimalarial effects comparable to that of existing antimalarial drugs. Nevertheless, the possibility exists of developing therapeutically active pharmacophores from phytochemical precursors. In addition to a compound's antimalarial activity its selectivity toward the parasite and safety to individuals infected with malaria are also of major importance [19]. Several studies fail to assess the time-dependent effects of pharmacologically active antimalarial principles derived from phytochemicals on the progression of various stages of the plasmodium parasite's reproductive cycle within erythrocytes or incidence of morphologic and dimensional changes on different stages of the parasite [20].

This study screened the anti-plasmodial activity of AG at different time points during the intraerythrocytic cycle of $P$. falciparum 3D7 while simultaneously determining its impact on the development of different stages of the parasite, its morphology, and related dimensional characteristics. AG has previously been reported to exert antiplasmodial activity against $P$. falciparum 3D7 [20, 21]. Furthermore, Megantara et al., (2015) reported the promising potential of $A G$ as an effective pharmacophore for developing new antimalarial drugs [21] .

In this experiment, the pure compound of $A G$ showed a good anti-plasmodial activity $\left(\mathrm{IC}_{50}=4148.61 \mathrm{nM}\right)$. However, the anti-plasmodial activity of AG was about 1000 -fold less than that of chloroquine. This suggests that further structural modifications could possibly be beneficial towards improving its anti-plasmodial activity. Furthermore, the results obtained corroborate previous observations reported by Zaid et al., [11]. The antiplasmodial activity of AG was attributed to its inhibitory effect against plasmepsins I, II, and IV by Megantara et al., (2015) [21]. Plasmepsin is an aspartate protease enzyme present abundantly in the acidic digestive vacuoles of Plasmodium [22]. It takes part in the degradative pathway that breaks down globin into essential amino acids. It is well known that Plasmodium relies on haemoglobin as its sole source of protein [23]. Haemoglobin is broken down by hemoglobinase enzymes into globin and heme, where the former is subjected to a series of degradative aspartate proteases to be broken down into its essential amino acids [24]. This pathway was also suggested as a candidate target for drugs used in malaria chemotherapy [24].

It is well known that CQ produces its effect by targeting the haemoglobin degradation pathway. Its mechanism of action is slightly different from that proposed for AG. It interferes with pathways that detoxify heme, an obnoxious waste product that is released after the breakdown of haemoglobin. It is detoxified inside the digestive vacuoles of 
plasmodia into hemozoin; an innocuous inert waste product of heme. Consequently, inhibition of heme detoxification results in its accumulation and induction of a cascade of heme-induced oxidative pathways [24].

In Plasmodium, haemoglobin degradation pathway starts during the ring stage and continues throughout early and mature trophozoite development. Its activity reaches its utmost level during the early trophozoite stage within the period of 6 to $12 \mathrm{~h}$ after cultivation of the synchronized ring stage [25].

The results showed that most of the parasiticidal effect due to chloroquine was observed within the first $12 \mathrm{~h}$ of the cycle wherein the ring stage predominated (Figs. 1 and 2). This finding is in good agreement with a report by Zhang (1986) [26] describing the time-dependent effect of CQ during the course of the intraerythrocytic cycle. Furthermore, CQ produced noticeable morphological changes during this period on both rings and trophozoites. These changes were characterized by cellular shrinkage, cellular disfiguration, and loss of chromatin materials (Fig. 3). In addition, morphological changes were absent at the schizont stage in agreement with reports by Zhang et al., (1986). Overall, morphological changes were observable and persisted up to the end of the cycle indicating that CQ compromised the cellular activity of the mature trophozoites without inducing prominent lethality.

The stage wherein breakdown of haemoglobin and release of heme commences is still being debated. Some reports have pointed out to the possible incidence of this pathway during the ring stage [26], while its maximum incidence occurs during early trophozoite development [25]. This suggests that the maximum effect of CQ should occur during the trophozoite, not the ring stage. The study contradicts this notion as it emphasizes that a prominent anti-plasmodium effect for CQ can be observed before the trophozoite stage and during the ring stage. Nevertheless, there are studies reporting that inhibition of heme detoxification and release of heme is not the only mechanism of anti-plasmodial activity of CQ. Some studies declared that CQ might interfere with the integrity of lysosomal membranes resulting in the increased release of degrading enzymes like caspase [27]. This, in turn, may affect the mitochondrial membrane and increase the release of cyt $\mathrm{C}$, which in turns triggers cellular apoptosis [28].

Andrographolide failed to produce similar changes as compared to CQ during the first $12 \mathrm{~h}$. Its impact was however notable after $12 \mathrm{~h}$ of the cycle, wherein dead parasites and morphologic changes in intact cells were observed (Figs. 3 and 4). However, these changes were less prominent as compared to those induced by CQ. This is because $A G$ failed to produce noticeable morphological changes during the early stages of the parasite inoculation and did not exhibit any effect on cellular viability during the late stages of inoculation. Also, the delayed lethality can be attributed to its impact on targets that showed their utmost expression during this stage. For instance, AG was suggested as a potent inhibitor of the plasmepsin enzyme [21], which is involved in haemoglobin breakdown [29].

Andrographolide is derived from a labdane diterpenoid (a terpene derivative), Goulart et al., (2004) elucidated that some terpene compounds (nerolidol, farnesol, and linalool) show inhibitory effect(s) on the isoprenoid biosynthesis of trophozoite and schizont of $P$. falciparum [30]. Furthermore, the mono-terpenes have been reported to inhibit the growth of $P$. falciparum by inhibition of protein prenylation [31]. From these, it was surmised that AG might have exerted a negative impact on the functional characters of isoprenoid synthesis pathway, which begins in apicoplasts. Apicoplasts are relict organelles that occur as parts of the cellular structure of apicomplexan protozoa. They (apicoplasts) possess several functions ranging from pathways inavolved in fatty acid, isoprenoid, iron-sulphur, and heam biosynthesis [32]. The isoprenoid synthesis pathway is considered the most important 
pathway within mature trophozoites and can be set as an essential drug target for malaria chemotherapy [30]. This pathway is essential, as it fuels the cell with the required energy [33].

After commencement of the synchronized cycle, the results showed that the number of viable parasites subsequent to CQ exposure was decreased compare to the control, moreover, this number remained the same after $24 \mathrm{~h}$ as compared to that after $12 \mathrm{~h}$. In the drug sensitivity assay, only a small decline in the inhibitory growth parameters was observed. This was attributed to the pLDH assay being performed at the same time as the drug sensitivity assay, which overestimates the parasitemia when measuring the enzyme activity of non-viable parasites. The number of viable cells decreased prominently as per the results of the Giemsa-stained thin blood film, but the changes in the growth inhibitory parameters as per the results of the sensitivity assay were less. The pLDH enzyme is involved in the conversion of lactate to pyruvate, and helps in the release of energy in the form of Adenosine Triphosphate ATP. Its activity may persist even after parasite death, and is responsible for the overestimation of parasite growth while using the well-known pLDH technique [34, 35], unlike SYBR Green I Malaria Drug Sensitivity Assay: which detect the existence of malaria DNA of the parasite inside infected erythrocytes [36].

A similar situation was observed subsequent to AG exposure. However, in this case, dead parasites were absent during the first $12 \mathrm{~h}$. whereas, the dead parasites started to appear after $24 \mathrm{~h}$ of drug exposure. This implies that the anti-plasmodial activity of AG began $12 \mathrm{~h}$ after commencement of the cycle. Similarly, inhibitory growth parameters due to $A G$ were prominently lesser after $48 \mathrm{~h}$ as compared to that at $24 \mathrm{~h}$. However, results of Giemsastained thin blood smears for AG treated flasks showed a comparable number of the parasites after 24 and $48 \mathrm{~h}$. The morphological features of the cells exposed to CQ suggested the incidence of necrosis and apoptosis within the parasites. Meanwhile, the death crisis features of the AG-treated flasks were suggestive of a mild form of apoptosis. The incidence of apoptosis is expected when the mitochondrial membrane starts losing its integrity, resulting in the release of cytochrome $\mathrm{C}$ and other apoptosis initiators into the cytosol. This, in turn, induces a cascade of the apoptotic pathway that is characterized by the activation of caspase enzymes and loss of cellular content [37]. Induction of the apoptosis pathway was detected for AG in most cancer mammalian cells models [38]. In addition to that, Luo et al., (2013) mentioned that during treatment of lung cancer cells, the nuclear transcription factor-kappa B (NF-kB) targets inhibition using AG [39]. However, further studies are warranted to elucidate the role of $A G$ in apoptotic pathways in the context of malaria.

\section{Conclusions}

In conclusion, this study suggests that the initial effect of $A G$ is exerted against mature trophozoites during the 12-24 $\mathrm{h}$ period after commencement of the intraerythrocytic cycle. However, the effect of CQ was more prominent as compared to $A G$, as observed from the rings and early trophozoites within the first $12 \mathrm{~h}$ of the cycle. Further investigations on the molecular mechanisms underlying the effect of AG on the Plasmodium parasite are warranted to better understand the time course of its anti-plasmodial activity on blood stage parasites and explore its potential application as an adjuvant to existing antimalarial therapy.

\section{Abbreviations}

AG

Andrographolide; ANOVA:Analysis of Variance; CQ:chloroquine; DMSO:Di-methylsulphoxide; $\mathrm{IC}_{20}$ :inhibitory concentration at $20 \%$ of maximal growth; $I_{50}$ :inhibitory concentration at $50 \%$ of maximal growth; $I_{90}$ :inhibitory concentration at $90 \%$ of maximal growth; NBT:Nitroblue tetrazolium chloride; P. falciparum:Plasmodium 
falciparum; PBS:Phosphate buffer saline; PES:phenazine ethosulfate; pLDH:Plasmodial lactic dehydrogenase enzyme; PRBCs:Parasitized red blood cells; RBCs:Red blood cells; SEM:standard error of the mean; WHO:World Health Organization;

\section{Declarations}

\section{Acknowledgments}

The team acknowledges the Faculty of Medicine and Health Sciences, University Putra Malaysia, for providing the required facilities and equipment to run the experiment.

Author Contributions:

Conceptualization of ideas, A.A.I.A., R.A.M., and Z.O.I; experimental design, A.A.I.A., Z.O.l; article preparation, A.A.I.A., and Z.O.I; technical assistance and reviewed the findings, S.A.A., and M.N.W; revision of the manuscript content and editing, R.B., I.S.I., R.O.B., N.Z.U; final approval, all authors have read and agree to the published version of the manuscript.

Funding

This research received funding from the Ministry of Higher Education, Malaysia, by supporting this work through the Research Grant number: 5524777. University Putra Malaysia.

Availability of data and materials

The datasets used and/or analysed during the current study are available from the corresponding author on reasonable request.

Ethics approval and consent to participate

approval was waived

Consent for publication

Not applicable.

Disclosure

Some aspects of this work were presented as abstract at INFECTIONS 2017 conference at Universiti Putra Malaysia, as well as the Annual Scientific Conference of Malaysian Society of Parasitology and Tropical Medicine, March 2018.

Competing interests

The authors declare that they have no competing interests.

\section{References}

1. WHO. World Malaria Report. https://www.who.int/malaria. 2018. p. (accessed 23 Sep 2019). 
2. Cox FE. History of the discovery of the malaria parasites and their vectors. Parasit Vectors. 2010;3,1:1-9.

3. Warhurst DC. Antimalarial Drugs. Drugs [Internet]. 1987;33(1):50-65.

4. Gelband, H., Panosian, C. B., \& Arrow KJE. Saving Lives, Buying Time: Economics of Malaria Drugs in an Age of Resistance. Washington: THE NATIONAL ACADEMIES PRESS; 2004.

5. White NJ. Determinants of relapse periodicity in Plasmodium vivax malaria. Malar J. 2011;10(1):297.

6. White NJ. The assessment of antimalarial drug efficacy. Trends Parasitol. 2002;18(10):458-64.

7. Niranjan A, Tewari SK, Lehri A. Biological activities of Kalmegh (Andrographis paniculata Nees) and its active principles-A review. Indian J Nat Prod Resour. 2010;1(2):125-35.

8. Wasman SQ, Mahmood AA, Chua LS, Alshawsh MA, Hamdan S. Antioxidant and gastroprotective activities of Andrographis paniculata (Hempedu Bumi) in Sprague Dawley rats. Indian J Exp Biol. 2011;49(10):767-72.

9. Krithika R, Verma RJ, Shrivastav PS. Antioxidative and cytoprotective effects of andrographolide against CCl4induced hepatotoxicity in HepG2 cells. Hum Exp Toxicol. 2013;32(5):530-43.

10. Mishra, K., Dash, A. P., \& Dey N. Andrographolide: A novel antimalarial diterpene lactone compound from Andrographis paniculata and its interaction with curcumin and artesunate. J Trop Med. 2011;2011:1-6.

11. Zaid OI, Abd Majid R, Sabariah MN, Hasidah MS, Al-Zihiry K, Yam MF, et al. Andrographolide effect on both Plasmodium falciparum infected and non infected RBCs membranes. Asian Pac J Trop Med [Internet]. 2015;8(7):507-12. Available from: http://linkinghub.elsevier.com/retrieve/pii/S1995764515000644

12. Cranmer SL, Magowan C, Liang J, Coppel RL, Cooke BM. An alternative to serum for cultivation of Plasmodium falciparum in vitro. Trans R Soc Trop Med Hyg. 1997;91(3):363-5.

13. Trager W, Jensen JB. Human malaria parasites in continuous culture. Science (80- ) [Internet]. 1976;193(4254):673-5. Available from: http://www.ncbi.nlm.nih.gov/pubmed/781840

14. Makler MT, Ries JM, Williams JA, Bancroft JE, Piper RC, Gibbins BL HD. Parasite lactate dehydrogenase as an assay for Plasmodium falciparum drug sensitivity. Am J Trop Med Hyg. 1993;48(6):739-741.

15. Lambros C, Vanderberg JP. Synchronization of Plasmodium falciparum Erythrocytic Stages in Culture. J Parasitol. 1979;65(3):418-20.

16. WHO. World Health Organization. Basic malaria microscopy. Parasitol Today. 2010;2nd Learne(Part1.).

17. Tu Y. The discovery of artemisinin (qinghaosu) and gifts from Chinese medicineo Title. Nat Med. 2011;17(10):1217-20.

18. WHO. World Malaria Report. https://www.who.int/malaria. 2015. p. (accessed 15 Dec 2017).

19. Kaur K, Jain M, Kaur T, Jain R. Antimalarials from nature. Bioorg Med Chem. 2009 May 1;17(9):3229-56.

20. Derbyshire ER, Mota MM, Clardy J. The next opportunity in anti-malaria drug discovery: The liver stage. PLoS Pathog. 2011;7(9).

21. Megantara S, Levita J, Surantaatmadja SI. In Silico Study of Andrographolide as Protease Inhibitors for Antimalarial Drug Discovery BT - 3rd International Conference on Computation for Science and Technology (ICCST-3). In: In 3rd International Conference on Computation for Science and Technology (ICCST-3) Atlantis Press [Internet]. Atlantis Press; 2015. p. 36-9. Available from: https://doi.org/10.2991/iccst-15.2015.8

22. Eggleson, K. K., Duffin, K. L., \& Goldberg DE. Identification and characterization of falcilysin, a metallopeptidase involved in haemoglobin catabolism within the malaria parasite Plasmodium falciparum. J Biol Chem. 1999;274(45):32411-7. 
23. Graham H. Coombs, Daniel E. Goldberg, Michael Klemba, Colin Berry, John Kay JCM. Aspartic proteases of Plasmodium falciparum and other parasitic protozoa as drug targets. Trends Parasitol. 2001;17(11):532-7.

24. Francis SE, Sullivan DJ, Goldberg DE. Hemoglobin metabolism in the malaria parasite Plasmodium falciparum. Annu Rev Microbiol. 1997;51:97-123.

25. Avner Yayon, John A. Vande Waa, Malka Yayon, Timothy G. Geary AJBJ. Stage-Dependent Effects of Chloroquine on Plasmodium falciparum In Vitro. JPorozool. 1983;30(4):642-7.

26. Ya Zhang KS. O. A and AJ. Stage-Dependent Inhibition of Chloroquine on Plasmodium falciparum In vitro. J Paras, Am Soc Parasitol Stable. 1986;72(6):830-6.

27. Geng Y, Kohli L, Klocke BJ, Roth KA. Chloroquine-induced autophagic vacuole accumulation and cell death in glioma cells is p53 independent. Neuro Oncol. 2010;12(5):473-81.

28. Picot S, Burnod J, Bracchi V, Chumpitazi BFF, Ambroise-Thomas P. Apoptosis related to chloroquine sensitivity of the human malaria parasite Plasmodium falciparum. Trans R Soc Trop Med Hyg. 1997;91(5):590-1.

29. Francis SE, Banerjee R, Goldberg DE. Biosynthesis and Maturation of the Malaria Aspartic Hemoglobinases Plasmepsins I and II *. J Biol Chem. 1997;272(23):14961-8.

30. Goulart HR, Kimura EA, Peres VJ, Couto AS, Duarte FAA, Katzin AM. Terpenes arrest parasite development and inhibit biosynthesis of isoprenoids in Plasmodium falciparum. Antimicrob Agents Chemother. 2004;48(7):2502-9.

31. Moura IC, Wunderlich G, Uhrig ML, Couto AS, Peres VJ, Katzin AM, et al. Limonene arrests parasite development and inhibits isoprenylation of proteins in Plasmodium falciparum. Antimicrob Agents Chemother. 2001;45(9):2553-8.

32. Waller, R. F., \& McFadden GI. The Apicoplast: A Review of the Derived Plastid of Apicomplexan Parasites. Curr Issues Mol Biol. 2005;7:57-80.

33. Guggisberg AM, Amthor RE, Odom AR. Isoprenoid biosynthesis in Plasmodium falciparum. Eukaryot Cell. 2014;13(11):1348-135.

34. Verma P, Biswas S, Mohan T, Ali S, Rao DN. Detection of histidine rich protein \& lactate dehydrogenase of Plasmodium falciparum in malaria patients by sandwich ELISA using in-house reagents. Indian J Med Res. 2013;138(6):977-87.

35. Krampa F, Aniweh Y, Awandare G, Kanyong P. Recent Progress in the Development of Diagnostic Tests for Malaria. Diagnostics. 2017;7(3):54.

36. Vossen MG, Pferschy S, Chiba P, Noedl H. The SYBR green I malaria drug sensitivity assay: Performance in low parasitemia samples. Am J Trop Med Hyg. 2010;82(3):398-401.

37. Elmore S. Apoptosis: A Review of Programmed Cell Death. Toxicol Pathol. 2007;35(4):495-516.

38. Lim JCW, Chan TK, Ng DS, Sagineedu SR, Stanslas J, Wong WF. Andrographolide and its analogues: Versatile bioactive molecules for combating inflammation and cancer. Clin Exp Pharmacol Physiol. 2012;39(3):300-10.

39. Luo W, Liu Y, Zhang JUN, Luo X, Lin C, Guo J. Andrographolide inhibits the activation of NF - K B and MMP - 9 activity in H3255 lung cancer cells. Exp Ther Med. 2013;6(3):743-6.

\section{Figures}




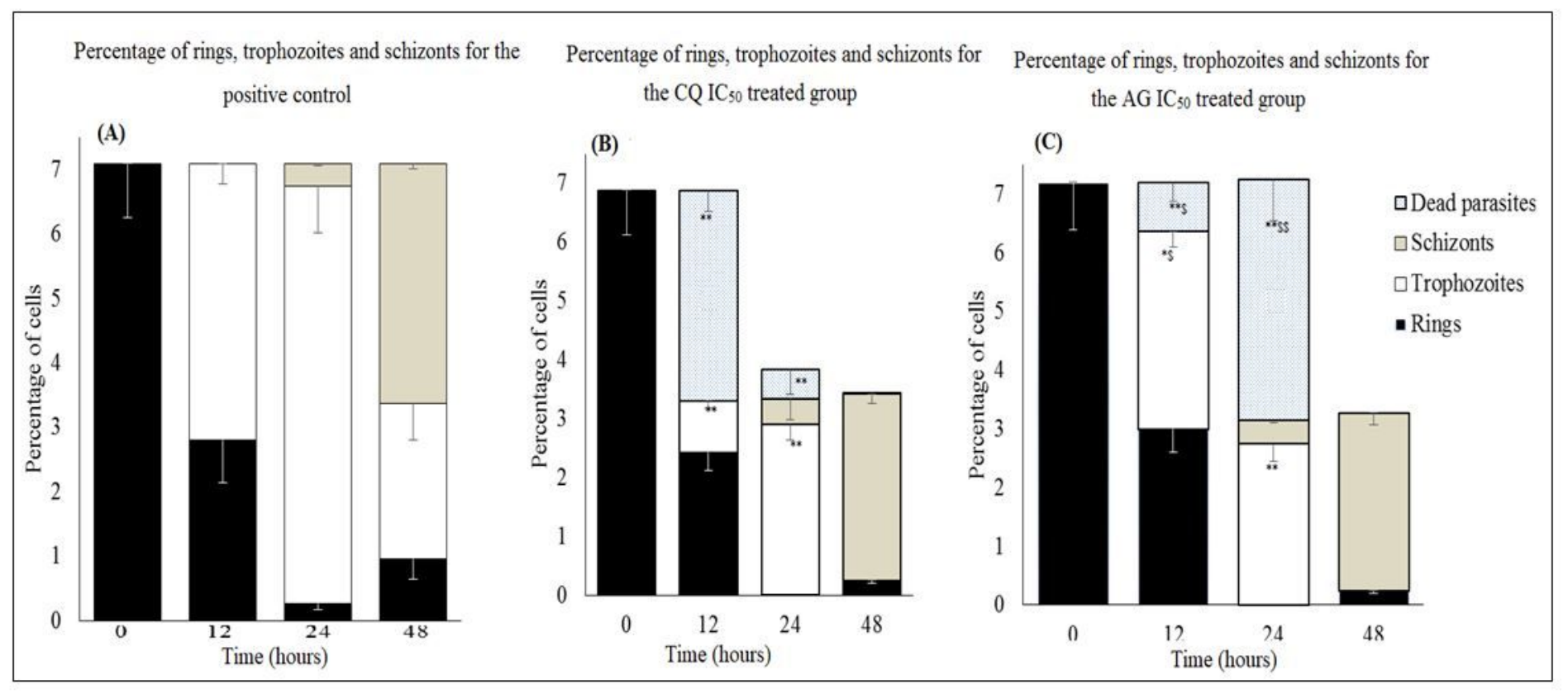

\section{Figure 1}

Cell cycle progression of P. falciparum 3D7 cultivated in untreated as control (A), CQ IC50 (B) and AG IC50 (C) treated culture flasks, respectively. CQ IC50 and AG IC50 stand for the concentrations of CQ and AG required to inhibit the parasite growth by $50 \%$ as determined by the drug sensitivity assay, respectively (Table 1, Figure 1). The results were expressed as mean \pm SEM value of the percentage of parasitemia of each stage of the intraerythrocytic cycle versus time in an hour. ${ }^{*}, * \star$ and $\$, \$ \$$ represent statistically significant difference as compared to control and chloroquine with $\mathrm{P}<0.01$ and $\mathrm{P}<0.05$ respectively.

\begin{tabular}{|l|l|l|l|}
\hline Drugs exposure & Ring stage at 12 hours & Trophozoite stage at 24 hours & Schizont stage at 48 hours \\
\hline Control & & & \\
\hline
\end{tabular}

Figure 2 
Giemsa stained thin blood films showing the morphological changes at different stages of the intraerythrocytic cycle of P. falciparum 3D7 in the non-exposure control, CQ and AG following exposure to their IC50 concentration at different time intervals (12, 24 and 48 hours) taken at objective 100X. The orange arrows indicate changes in the appearance of the exposed parasites including prominent shrinkage, fragmented structure, and loss of cellular integrity.
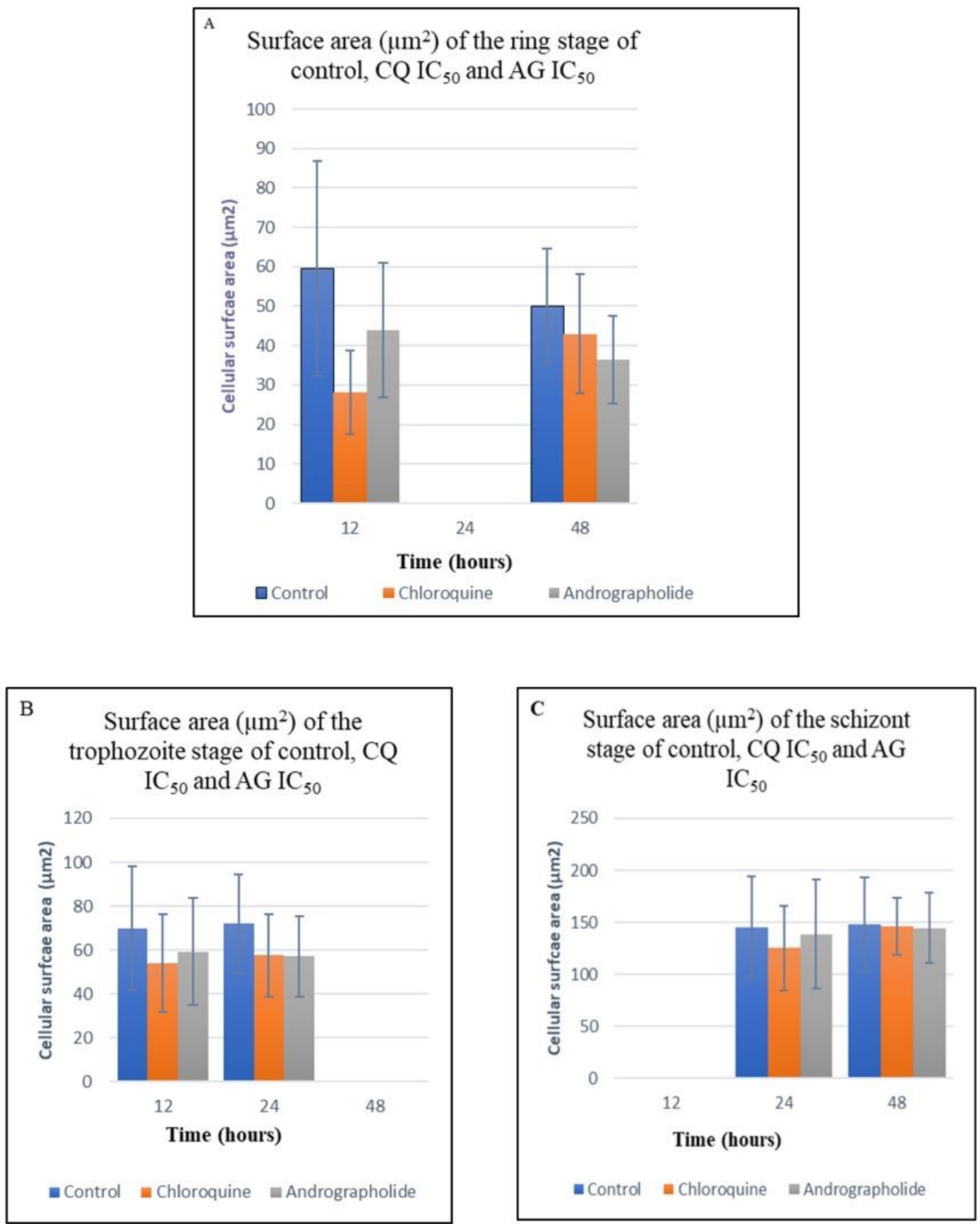

\section{Figure 3}

The distribution of mean diameter changes of P. falciparum 3D7 following exposure to AG and CQ after 12, 24 and 48 hours (Figures $\mathrm{A}$ at the ring stage, $\mathrm{B}$ at the trophozoite stage and $\mathrm{C}$ at the schizont stage, respectively). 